\title{
Resonant demagnetization of a dipolar Bose-Einstein condensate in a three-dimensional optical lattice
}

\author{
A. de Paz, ${ }^{1,2}$ A. Chotia, ${ }^{1,2}$ E. Maréchal, ${ }^{1,2}$ P. Pedri, ${ }^{1,2}$ L. Vernac, ${ }^{1,2}$ O. Gorceix, ${ }^{1,2}$ and B. Laburthe-Tolra ${ }^{1,2}$ \\ ${ }^{1}$ Laboratoire de Physique des Lasers, Université Paris 13, Sorbonne Paris Cité, F-93430 Villetaneuse, France \\ ${ }^{2}$ LPL, CNRS, UMR 7538, F-93430 Villetaneuse, France
}

(Received 20 December 2012; revised manuscript received 26 March 2013; published 31 May 2013)

\begin{abstract}
We study dipolar relaxation of a chromium Bose-Einstein condensate loaded into a three-dimensional (3D) optical lattice. We observe dipolar relaxation resonances when the magnetic energy released during the inelastic collision matches an excitation towards higher-energy bands. Spectroscopy of these resonances for two orientations of the magnetic field provides a 3D band spectroscopy of the lattice. The narrowest resonance is registered for the lowest excitation energy. Its line shape is sensitive to the on-site interaction energy. We use such sensitivity to probe number squeezing in a Mott insulator.
\end{abstract}

DOI: 10.1103/PhysRevA.87.051609

PACS number(s): 67.85.Hj, 03.75.Mn, 37.10.Jk

Quantum dipolar gases have attracted much attention in recent years [1-3]. Following the seminal studies of Cr BoseEinstein condensates (BECs) [4-7] and the recent production of Er [8] and Dy [9,10] quantum gases, it is natural to study dipolar gases confined in optical lattices. Dipolar gases in lattices provide an ideal playground for studying quantum phase transitions in a system with long-range interactions. The unique properties of dipole-dipole interactions (DDIs) present direct similarities with the Heisenberg model of quantum magnetism [11-14] and lead to novel quantum phases displaying possible long-range ordering in lattices [15-17]. The nonlinear coupling between spin and orbital degrees of freedom provided by dipolar interactions [18-23] is particularly interesting. Although at large magnetic field, this coupling leads to fast dipolar relaxation losses for atoms in excited spin states [24], we have recently demonstrated that working at low magnetic field enables the study of multicomponent quantum gases with free magnetization $[25,26]$. Here we extend this research to include dipolar particles trapped in optical lattices and directly observe discrete, atom-number-dependent coupling between spin and orbital degrees of freedom.

We study the magnetization dynamics of ${ }^{52} \mathrm{Cr}$ atoms loaded in a deep three-dimensional (3D) optical lattice. Under our experimental conditions, we produce a Mott insulator state with a core of two particles per site [27,28]. Compared to our previous results in two-dimensional (2D) optical lattices [23], the 3D confinement allows us to reach a new regime, where on-site dipolar relaxation is inhibited unless the released magnetic energy matches a lattice band excitation: dipolar relaxation is a resonant process. The interplay between the anisotropies of dipolar interaction and of lattice sites leads to a resonance spectrum which depends on the magnetic-field orientation. Measuring demagnetization as a function of the magnetic field for two orientations allows for spectroscopy of the 3D lattice band structure. We operate in an asymmetric $3 \mathrm{D}$ lattice such that the narrowest resonance is found to be sensitive to the on-site atom-number distribution and reveals the number-squeezed distribution of the Mott state. Changing our experimental conditions, we prepare sites containing three atoms. Spin-orbit coupling DDI then produces three-body states that are expected to have entangled spin and orbital degrees of freedom.
We produce a ${ }^{52} \mathrm{Cr}$ BEC (with spin $S=3$ ) with typically $N=1.5 \times 10^{4}$ atoms by forced evaporation in the lowestenergy state $\left|m_{S}=-3\right\rangle$ in a crossed optical dipole trap derived from a fiber laser at $1075 \mathrm{~nm}$ [29] $\left(m_{S}\right.$ is the spin projection along the magnetic field). The BEC is loaded adiabatically in a 3D optical lattice, generated using $4 \mathrm{~W}$ of a single-mode laser at $\lambda=532 \mathrm{~nm}$. A $2 \mathrm{D}$ horizontal lattice is created by the interference of three beams: two beams are counterpropagating, and the third intersects the retroreflected pair at $45^{\circ}$. The resulting $2 \mathrm{D}$ lattice is rectangular with a periodicity of $\lambda /\left(2 \cos \frac{\pi}{8}\right)$ along the $O x$ direction and $\lambda /\left(2 \sin \frac{\pi}{8}\right)$ along the $O y$ direction. In the vertical direction an independent 1D lattice is created by a vertical retroreflected beam with horizontal polarization. The $1 / e^{2}$ beams' radii at the BEC position are $60(10) \mu \mathrm{m}$. The lattice depth is measured by Kapitza-Dirac diffraction [30] to be $25 E_{R}$ (with $E_{R}=h^{2} / 2 m \lambda^{2}$ being the recoil energy), corresponding to first band excitation frequencies of $\omega_{x} / 2 \pi=170(10) \mathrm{kHz}$, $\omega_{y} / 2 \pi=55(5) \mathrm{kHz}$, and $\omega_{z} / 2 \pi=100(10) \mathrm{kHz}$.

For most of the experiments presented here, we load the optical lattice adiabatically in $40 \mathrm{~ms}$. Although the lattice geometry is complicated by its anisotropy and we do not expect a fully adiabatic crossing towards the Mott state, this loading time scale allows us to remain close to the ground state. This is confirmed by adiabatically ramping down the lattice at the same speed, in which case a BEC with only few excitations is recovered. For our experimental parameters, the predicted Mott state is made of a central region with double occupancy surrounded by a single-occupancy shell.

Once the lattice is loaded, atoms are adiabatically transferred from $\left|m_{S}=-3\right\rangle \equiv|-3\rangle$ to the highest-energy state $|3\rangle$ by means of a 5-ms rf sweep (the efficiency of rf sweeps is $85 \%$ ). Once atoms are transferred to $|3\rangle$, dipolar relaxation is allowed. After a given time, the magnetization is again reversed using a second rf sweep (this step is performed only to optimize the detection), the optical lattice is switched off in $1 \mathrm{~ms}$, and the populations in the different magnetic states are monitored by a Stern Gerlach analysis [Fig. 1(b)].

We obtain two different behaviors as a function of the magnetic-field amplitude $B$, i.e., whether the Larmor frequency $\hbar \omega_{\text {Lar }}=g_{S} \mu_{B} B$ is smaller or larger than the lattice depth $V_{0}\left(\mu_{B}\right.$ is the Bohr magneton, and $g_{S}=2$ is the Landé 


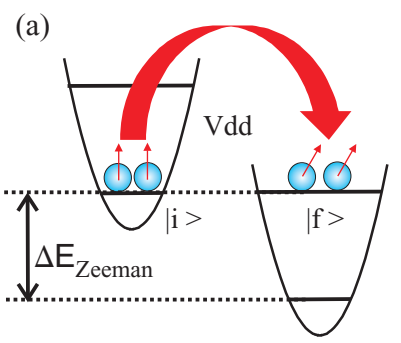

(b)

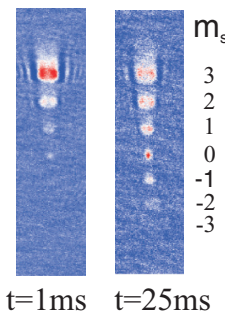

FIG. 1. (Color online) (a) Sketch of dipolar relaxation, which only occurs provided the loss of Zeeman energy $\Delta E_{\text {Zeeman }}$ matches a gain in orbital energy (band excitation). (b) State composition at resonance after $6 \mathrm{~ms}$ and after $30 \mathrm{~ms}$ of dipolar relaxation (absorption images after Stern-Gerlach separation).

factor). For large magnetic fields, $\hbar \omega_{\text {Lar }}>V_{0}$, we observe a nonexponential loss dynamics: the atom number rapidly decreases before reaching a nonzero steady value, and then no losses are observed for tens of milliseconds. Losses are due to dipolar relaxation between atoms occupying the same site, and they stop when only single-occupancy sites remain.

At low magnetic field, $\hbar \omega_{\text {Lar }} \ll V_{0}$, no losses are observed, as atoms remain trapped after dipolar relaxation. We instead observe a demagnetization dynamics that reveals a transfer of atoms from $|3\rangle$ to $|2\rangle$ and then to lower-energy magnetic states [Fig. 1(b)]. For a fixed relaxation time we registered the final population in $|3\rangle$ as a function of the magnetic-field amplitude. The spectra presented in Fig. 2 show resonance peaks which we interpret as the consequence of total-energy conservation: for a pair of atoms initially in the vibrational ground state of one lattice site, spin relaxation only occurs if the released magnetic energy matches a band excitation. We obtain two different spectra for two orthogonal directions of the magnetic field $\mathbf{B}$. This shows that dipolar relaxation couples the initial state to different lattice bands depending on the orientation of $\mathbf{B}$ relative to the geometry of a lattice site.

To interpret our data, we consider a simple theoretical model with two particles in a purely 3D harmonic anisotropic potential (with frequencies $\omega_{x}, \omega_{y}, \omega_{z}$ ), describing one lattice site. Dynamics is determined by the dipole-dipole interaction $V_{d d}(\mathbf{r})=\frac{d^{2}}{r^{5}}\left[r^{2} \mathbf{S}_{1} \cdot \mathbf{S}_{2}-3\left(\mathbf{S}_{1} \cdot \mathbf{r}\right)\left(\mathbf{S}_{2} \cdot \mathbf{r}\right)\right]$, where $d^{2}=\mu_{0}\left(g_{S} \mu_{B}\right)^{2} / 4 \pi, \mu_{0}$ is the magnetic constant, $\mathbf{r}=\mathbf{r}_{1}-$ $\mathbf{r}_{2}$ is the relative coordinate, and $\mathbf{S}_{i}$ is the spin operator for atom $i$. In the presence of an harmonic confinement, the center of mass and the relative motions are decoupled. DDIs affect only the relative motions; therefore only the relative coordinates need to be considered, with eigenstate $\left|n_{x}, n_{y}, n_{z}\right\rangle$. For the magnetic state of the pair, we use the molecular basis $\left|S_{M}, m_{M}\right\rangle$ where $S_{M}$ is the total spin, and $m_{M}$ is the spin projection. The energy is given by $E_{\mathbf{n}, m_{M}}=\sum_{i} \hbar \omega_{i}\left(n_{i}+\frac{1}{2}\right)+m_{M} g_{S} \mu_{B} B$, where $i=x, y, z$. The particles are prepared in the initial state $|i\rangle=|0,0,0\rangle \otimes|6,6\rangle$.

From this initial state, due to DDIs, three spinrelaxation channels are possible with final spin states $|6,5\rangle,|6,4\rangle$, and $|4,4\rangle$ [24]. The couplings to these three final states are described by the DDI coupling operators $V_{1}=3 S^{\frac{3}{2}} d^{2}\left(\hat{x}^{\prime}+i \hat{y}^{\prime}\right) \hat{z}^{\prime} / \hat{r}^{5}, \quad V_{2}=\frac{3}{2} \sqrt{\frac{6}{11}} S d^{2}\left(\hat{x}^{\prime}+\right.$ $\left.i \hat{y}^{\prime}\right)^{2} / \hat{r}^{5}$, and $V_{3}=\frac{3}{2} \sqrt{\frac{5}{11}} S d^{2}\left(\hat{x}^{\prime}+i \hat{y}^{\prime}\right)^{2} / \hat{r}^{5}$, where the orientation of the magnetic field $\mathbf{B}$ is along the $z^{\prime}$ axis [24] $\left(x^{\prime}, y^{\prime}\right.$, and $z^{\prime}$ are the relative coordinates). The first channel induces a loss of magnetic energy $g_{S} \mu_{B} B$, and the two others induce a loss of $2 g_{S} \mu_{B} B$.

As sketched in Fig. 1 the Zeeman energy is converted into excitation to a higher orbital state $\left|n_{x}, n_{y}, n_{z}\right\rangle$. Due to the dependence of $V_{1,2,3}$ on $x^{\prime}, y^{\prime}$, and $z^{\prime}$, the selection rules and the coupling strengths to the final orbital states strongly depend on the orientation of the magnetic field $\mathbf{B}$. This explains the difference between the two registered spectra (see Fig. 2) for $\mathbf{B}$ along $O x$ and $O y$, respectively. DDIs couple the initial orbital state with orbital excited states with even total symmetry. Additional specific selections rules are derived from the parity of $V_{1,2,3}$ in the three directions $x^{\prime}, y^{\prime}, z^{\prime}$. In practice, most of the resonances observed in Fig. 2 are attributed to channels 2 and 3. The observed resonances correspond to a rate of the order of $100 \mathrm{~Hz}$, in good agreement with a calculation of the coupling strengths $\left\langle i\left|V_{1,2,3}\right| f\right\rangle$. The relative coupling strengths are represented by the peak amplitudes in Fig. 2, and we also give the their value in the Supplemental Material [31].

A striking observation in Fig. 2 is that resonances widen as the excitation energy increases, so that they are well isolated

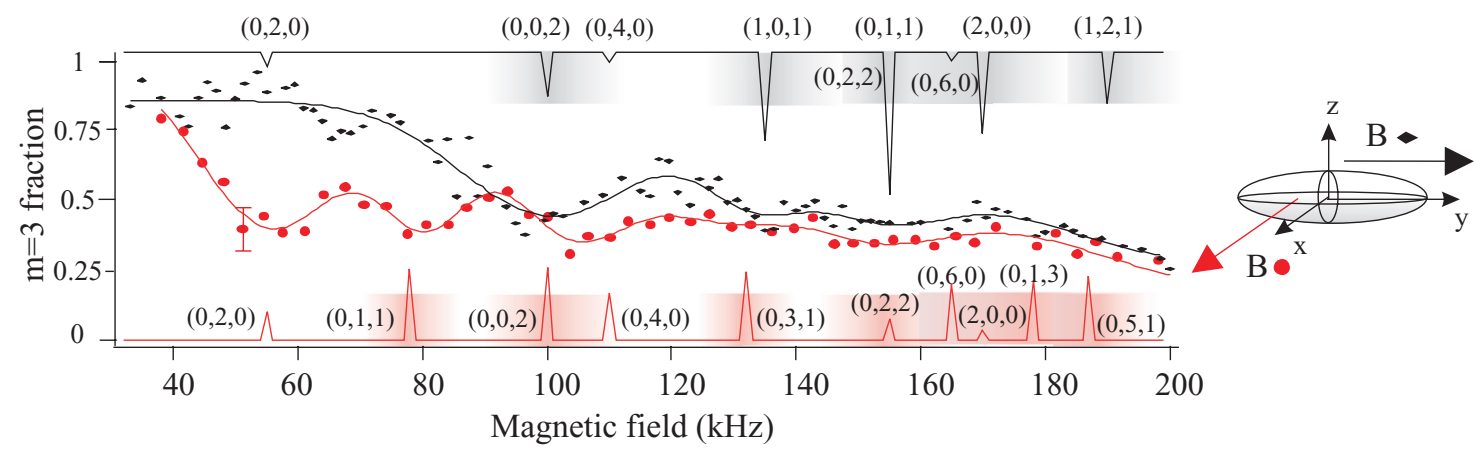

FIG. 2. (Color online) Observation of resonances due to dipolar relaxation as a function of the magnetic-field amplitude. The spectra (red dots: after $30 \mathrm{~ms}$ of dipolar relaxation time; black diamonds: after $20 \mathrm{~ms}$ ) are obtained, respectively, for a magnetic field along $O x$ and $O y$, two orthogonal directions in the horizontal plane. The sketch on the right represents the two studied magnetic-field orientations referred to a lattice site. The peaks represent the frequency positions and the calculated coupling amplitudes of the resonances leading to excitations of lattice bands labeled by numbers $\left(n_{x}, n_{y}, n_{z}\right)$. Shaded areas qualitatively represent energy broadening due to tunneling in excited bands. 
only at low magnetic field. The main source of broadening is the increasing bandwidth for higher-energy lattices bands (represented by the shadows around the peaks in Fig. 2). Due to large lattice periodicity in the $y$ direction, the tunneling rate is small even for the second excited band corresponding to the lowest-energy resonance at $\omega_{\text {Lar }}=\omega_{y}=2 \pi \times 55 \mathrm{kHz}$, but it increases rapidly for excited bands in the other directions (as represented in Fig. 2). Magnetic field and lattice depth fluctuations are broadening the resonances at the $10-\mathrm{kHz}$ level for data shown in Fig. 2. Another cause of broadening is cascading dipolar processes corresponding to excitations to higher bands, whose energies are not equally spaced due to anharmonicity (at the $2-\mathrm{kHz}$ level). Cascading is indeed observed in our experiment in which states down to $\left|m_{S}=-2\right\rangle$ are produced at long times [Fig. 1(b)]. Inhomogeneous broadening of the band structure due to the finite size of the lattice beams is of the order of $500 \mathrm{~Hz}$.

The lowest-frequency resonance has a small broadening due to tunneling $(100 \mathrm{~Hz})$, which potentially allows us to reveal the role of on-site interactions. This resonance occurs at $\omega_{\text {Lar }}=$ $\omega_{y}$, corresponding to an orbital excitation of 2 units $\left(2 \hbar \omega_{y}\right)$ and a change in Zeeman energy of $2 g_{S} \mu_{B} B$ (the coupling operators

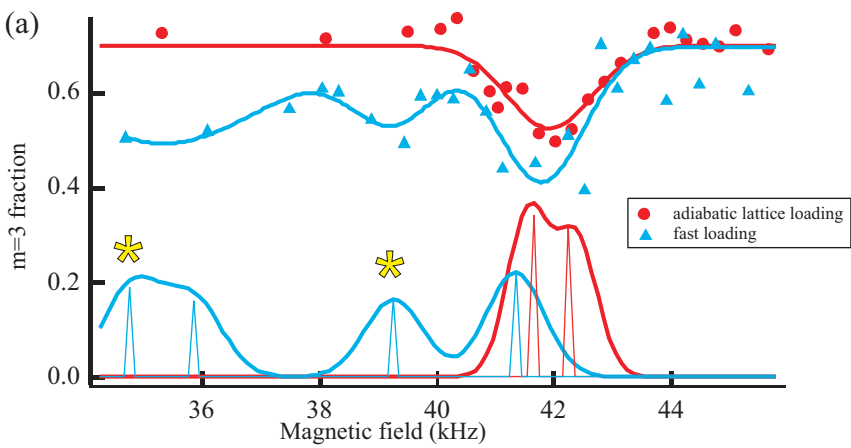

(b)

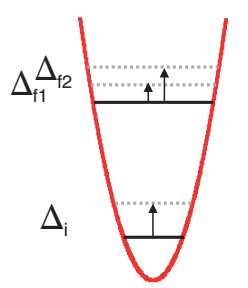

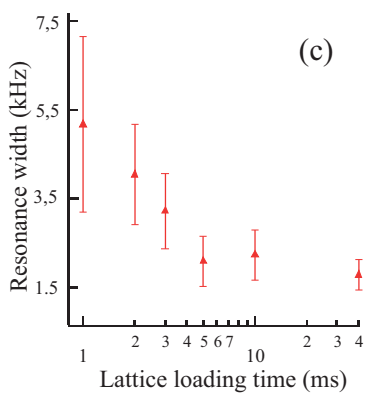

FIG. 3. (Color online) High-resolution spectroscopy of the lowest-frequency dipolar resonance: sensitivity to site atom number. (a) With an adiabatic loading (red dots), the line shape is narrow, while with a fast loading (blue triangles), a multiresonant structure appears. Each data point is the average of five measurements, and the lines are guides for the eyes. For the fast-loading case, this line is made using a fit with multiple Gaussians shifted from one another. On the bottom, we plot the calculated resonant peaks for doubly [red (dark gray) peak] and triply occupied sites [blue (light gray) peaks]. The stars indicate states with entangled spin and orbit. The bottom solid lines include an empirical broadening mimicking technical noise. (b) Sketch to interpret the site-occupancy sensitivity, arising from an on-site interaction shift depending on the orbital state in the lattice. (c) Resonance width (obtained by fitting the multiresonant spectra with a single Gaussian) as a function of the lattice loading time. are $V_{2}$ and $V_{3}$ ). In the rest of this Rapid Communication we focus on this resonance. For these measurements, shown in Fig. 3(a) after $11.5 \mathrm{~ms}$ of relaxation, the magnetic field and the laser intensity were actively stabilized to reduce technical broadening. These data were also registered at slightly lower lattice depth than for Fig. 2 data [corresponding to $\omega_{y}=45(1) \mathrm{kHz}$. To explain the observed data, the contact interactions $U\left(\mathbf{r}_{1}-\mathbf{r}_{2}\right)=U_{0} \delta\left(\mathbf{r}_{1}-\mathbf{r}_{2}\right)$ have to be taken into account $\left(U_{0}=\sum_{S_{t}=0,2,4,6}^{6} g_{S_{M}} P_{S_{M}}, P_{S_{M}}=\left|S_{M}\right\rangle\left\langle S_{M}\right|\right.$ is the total spin projector, and $g_{S_{M}}=4 \pi \hbar^{2} a_{S_{M}} / m$ and $a_{S_{M}}$ are the scattering lengths for the different molecular channels). The interactions shift the position of the resonances: for the lowest one we obtain $\omega_{\text {Lar }}=\omega_{y}+\frac{\Delta_{f}-\Delta_{i}}{2}$ [as sketched in Fig. 3(b)]. We calculate the interaction energy shifts of the initial and final states $\Delta_{i}$ and $\Delta_{f}$ using first-order perturbation theory assuming a harmonic trap.

For two particles in the same site, the on-site energy shift of the initial state $|i\rangle$ is given by $\Delta_{i}=\Delta_{0}$, where $\Delta_{0}=$ $(m / h)^{\frac{3}{2}}\left(\omega_{x} \omega_{y} \omega_{z}\right)^{\frac{1}{2}} g_{6}$. The final states are $\left|f_{1}\right\rangle=|0,2,0\rangle \otimes$ $|6,4\rangle$ and $\left|f_{2}\right\rangle=|0,2,0\rangle \otimes|4,4\rangle$. The spin-dependent interactions lift the degeneracy, and the two final states' shifts are $\Delta_{f 1}=\Delta_{0} / 2$ and $\Delta_{f 2}=\left(g_{4} / g_{6}\right) \Delta_{0} / 2$.

As the initial state is more confined than the final ones, on-site interactions induce a global redshift of the resonance (about $2.5 \mathrm{kHz}$ ) and a splitting of the resonance into two peaks [separated by $0.85 \mathrm{kHz}$; see Fig. 3(a)] corresponding to two molecular potentials. We do not resolve the two resonances for two atoms per site, likely due to broadening associated with residual magnetic-field fluctuations and lattice inhomogeneities.

In another set of measurements, we have loaded the lattice faster (in less than $5 \mathrm{~ms}$ ), which results in a statistical distribution of lattice sites with different occupation numbers [32]. As shown in Fig. 3(c), the observed dipolar resonance strongly broadens when the loading time is short, which is a direct consequence of the average increased number of particles per site, leading to increased on-site interaction. We further observe [Fig. 3(a), blue triangles] that the line shape of the resonance is then completely modified. It presents a multiresonant structure that we interpret below as due to dipolar relaxation within sites containing three atoms. For atoms in stretched states, the on-site interaction shift scales with the number of pairs. However, this is not the case when the spin degree of freedom is released and the previous result with two atoms per site cannot be simply scaled by the number of pairs. For this specific case with three atoms per site we will now show that DDIs couple the initial state to four different fully symmetric states, two of them having entangled spin and orbit. The observed multiresonant structure originates from these states.

To solve the case of three atoms per site, we have used the Jacobi coordinates: the orbital part of the threebody wave function can be described by the center-of-mass coordinate and two relative coordinates (see the Supplemental Material). As in the previous two-body problem, the centerof-mass motion is decoupled, and the total magnetization decreases by two units as the orbital energy increases by $2 \hbar \omega_{y}$. The initial state has maximal magnetization $M=9$, and each particle is in the ground state of the harmonic oscillator. The shift of this initial stretched state is $\Delta_{i}=3 \Delta_{0}$. 
We find four final bosonic states without center-of-mass excitation, a total magnetization $M=7$, and excitation energy of $2 \hbar \omega_{y}$. The shifts are given by the expressions $\Delta_{f 1}=(9 / 8) \Delta_{0}, \quad \Delta_{f 2}=(9 / 4) \Delta_{0}, \Delta_{f 3}=(a-b) \Delta_{0}$, and $\Delta_{f 4}=(a+b) \Delta_{0}$, with $a=\left(150 g_{4}+147 g_{6}\right) /\left(176 g_{6}\right)$ and $b=\left(5092 g_{4}^{2}+508 g_{4} g_{6}+4201 g_{6}^{2}\right)^{1 / 2} /\left(176 g_{6}\right)$. It can be noticed that, when $g_{4}=g_{6}$, only two resonances are expected.

Two of these four states are of the form $|O\rangle \otimes|S\rangle$, where $|O\rangle$ and $|S\rangle$ are symmetrical states of the orbital and spin parts, respectively. The two others are entangled states of orbital and spin degrees of freedom. Similar states produced by spinchanging collisions have also been discussed in the context of Fermi spinor gases [33].

The broadened resonance at fast loading signals that sites with more than two atoms are then statistically produced. This is further shown by the relatively good agreement between our theoretical model and the experimental data (see Fig. 3). This is an indication that three-body states with entangled spin and orbital degrees of freedom might be produced. This would enable interesting experiments where correlated tunneling of these exotic states could be studied.

These data are also relevant from the many-body point of view. Indeed, Fig. 3(a) also shows that, provided the lattice is loaded slowly, no sites with three atoms are produced. This is the indication that a squeezed atom distribution peculiar to the Mott state has been reached. On the other hand, for a short loading time sites with three atoms are produced [32]. In Fig. 3(c) we show the resulting increase of the resonance width for loading times shorter than $5 \mathrm{~ms}$. This adiabatic time scale is comparable to the trap period, which suggests that the breakdown of adiabaticity for fast loading arises from a time scale that is so short that the cloud does not have time to swell to reach the ground state of the system.

In conclusion, we have observed anisotropic dipolar resonances that couple the spin and the orbital degrees of freedom of bosons in a 3D lattice. We have focused on the narrowest resonance which could be used to engineer few-body entangled states and to probe the atom-number distribution in the Mott state. Dipolar resonances produce correlated states whose tunneling properties would be interesting to study. An interesting prospect would also be to reach the coherent regime. For example, starting from atoms in $|-2\rangle$, one might expect Rabi oscillations coupling states of different magnetization and different orbital states. Finally, we also stress that away from dipolar resonances, the magnetization is fixed, and the gas is stable. This has very important consequences, as this system is then a good candidate to study lattice magnetism at constant magnetization, with interesting connections to quantum magnetism [12-14,34]. The magnetic stability away from dipolar relaxation resonances displays similarities with the stability of quantum dots discussed in the context of quantum information computing [35].

LPL is Unite Mixte (UMR 7538) of CNRS and of Université Paris 13. We acknowledge financial support from Conseil Régional d'Ile-de-France and from Ministère de l'Enseignement Supérieur et de la Recherche under DIM NanoK/IFRAF and a CPER contract. A.C. thanks CNRS for financial support. We thank J. V. Porto and M. Gajda for their suggestions and their comments during the preparation of the manuscript.
[1] M. Baranov, L. Dobrek, K. Gòral, L. Santos, and M. Lewenstein, Phys. Scr. 2002, 74 (2002).

[2] M. Baranov, Phys. Rep. 464, 71 (2008).

[3] T. Lahaye, C. Menotti, L. Santos, M. Lewenstein, and T. Pfau, Rep. Prog. Phys. 72, 126401 (2009).

[4] J. Stuhler, A. Griesmaier, T. Koch, M. Fattori, T. Pfau, S. Giovanazzi, P. Pedri, and L. Santos, Phys. Rev. Lett. 95, 150406 (2005).

[5] T. Lahaye, J. Metz, B. Fröhlich, T. Koch, M. Meister, A. Griesmaier, T. Pfau, H. Saito, Y. Kawaguchi, and M. Ueda, Phys. Rev. Lett. 101, 080401 (2008).

[6] G. Bismut, B. Pasquiou, E. Maréchal, P. Pedri, L. Vernac, O. Gorceix, and B. Laburthe-Tolra, Phys. Rev. Lett. 105, 040404 (2010).

[7] G. Bismut, B. Laburthe-Tolra, E. Maréchal, P. Pedri, O. Gorceix, and L. Vernac, Phys. Rev. Lett. 109, 155302 (2012).

[8] K. Aikawa, A. Frisch, M. Mark, S. Baier, A. Rietzler, R. Grimm, and F. Ferlaino, Phys. Rev. Lett. 108, 210401 (2012).

[9] M. Lu, N. Q. Burdick, S. H. Youn, and B. L. Lev, Phys. Rev. Lett. 107, 190401 (2011).

[10] M. Lu, N. Q. Burdick, and B. L. Lev, Phys. Rev. Lett. 108, 215301 (2012).

[11] A. Auerbach, Interacting Electrons and Quantum Magnetism (Springer, New York, 1994), p. 255.
[12] A. Micheli, G. K. Brennen, and P. Zoller, Nat. Phys. 2, 341 (2006).

[13] A. V. Gorshkov, S. R. Manmana, G. Chen, J. Ye, E. Demler, M. D. Lukin, and A. M. Rey, Phys. Rev. Lett. 107, 115301 (2011).

[14] D. Peter, S. Müller, S. Wessel, and H. P. Büchler, Phys. Rev. Lett. 109, 025303 (2012).

[15] C. Menotti, C. Trefzger, and M. Lewenstein, Phys. Rev. Lett. 98, 235301 (2007).

[16] K. Góral, L. Santos, and M. Lewenstein, Phys. Rev. Lett. 88, 170406 (2002).

[17] E. G. Dalla Torre, E. Berg, and E. Altman, Phys. Rev. Lett. 97, 260401 (2006).

[18] Y. Kawaguchi, H. Saito, and M. Ueda, Phys. Rev. Lett. 96, 080405 (2006).

[19] L. Santos and T. Pfau, Phys. Rev. Lett. 96, 190404 (2006).

[20] K. Gawryluk, M. Brewczyk, K. Bongs, and M. Gajda, Phys. Rev. Lett. 99, 130401 (2007).

[21] B. Sun and L. You, Phys. Rev. Lett. 99, 150402 (2007).

[22] T. Świsłocki, T. Sowiński, J. Pietraszewicz, M. Brewczyk, M. Lewenstein, J. Zakrzewski, and M. Gajda, Phys. Rev. A 83, 063617 (2011).

[23] B. Pasquiou, G. Bismut, E. Maréchal, P. Pedri, L. Vernac, O. Gorceix, and B. Laburthe-Tolra, Phys. Rev. Lett. 106, 015301 (2011). 
[24] B. Pasquiou, G. Bismut, Q. Beaufils, A. Crubellier, E. Maréchal, P. Pedri, L. Vernac, O. Gorceix, and B. Laburthe-Tolra, Phys. Rev. A 81, 042716 (2010).

[25] B. Pasquiou, E. Maréchal, G. Bismut, P. Pedri, L. Vernac, O. Gorceix, and B. Laburthe-Tolra, Phys. Rev. Lett. 106, 255303 (2011).

[26] B. Pasquiou, E. Maréchal, L. Vernac, O. Gorceix, and B. Laburthe-Tolra, Phys. Rev. Lett. 108, 045307 (2012).

[27] D. Jaksch, C. Bruder, J. I. Cirac, C. W. Gardiner, and P. Zoller, Phys. Rev. Lett. 81, 3108 (1998).

[28] M. Greiner, O. Mandel, T. Esslinger, T. W. Hansch, and I. Bloch, Nature (London) 415, 39 (2002)

[29] Q. Beaufils, R. Chicireanu, T. Zanon, B. Laburthe-Tolra, E. Marechal, L. Vernac, J.-C. Keller, and O. Gorceix, Phys. Rev. A 77, 061601 (2008).
[30] P. L. Gould, G. A. Ruff, and D. E. Pritchard, Phys. Rev. Lett. 56, 827 (1986).

[31] See Supplemental Material at http://link.aps.org/supplemental/ 10.1103/PhysRevA.87.051609 for the values of the amplitudes of the resonances shown in Fig. 2, and for the calculation details of resonances for triply occupied sites shown in Fig. 3.

[32] M. Greiner, O. Mandel, T. W. Hansch, and I. Bloch, Nature (London) 419, 51 (2002).

[33] N. Bornemann, P. Hyllus, and L. Santos, Phys. Rev. Lett. 100, 205302 (2008).

[34] R. Barnett, D. Petrov, M. Lukin, and E. Demler, Phys. Rev. Lett. 96, 190401 (2006).

[35] M. Paillard, X. Marie, P. Renucci, T. Amand, A. Jbeli, and J. M. Gérard, Phys. Rev. Lett. 86, 1634 (2001). 